\title{
De novo and biallelic DEAF1 variants cause a phenotypic spectrum
}

\author{
Maria J. Nabais Sá, MD, PhD ${ }^{1}$, Philip J. Jensik, PhD², Stacey R. McGee, MS², Michael J. Parker, MD², \\ Nayana Lahiri, MD ${ }^{4}$, Evan P. McNeil, $\mathrm{MS}^{5}$, Hester Y. Kroes, MD, PhD ${ }^{6}$, Randi J. Hagerman, MD ${ }^{7,8}$, \\ Rachel E. Harrison, MD ${ }^{9}$, Tara Montgomery, MD ${ }^{10}$, Miranda Splitt, MD ${ }^{10}$, \\ Elizabeth E. Palmer, MBBS, BA ${ }^{11,12}$, Rani K. Sachdev, MD ${ }^{11,12}$, Heather C. Mefford, MD, PhD ${ }^{13}$, \\ Abbey A. Scott, BS, MS ${ }^{14}$, Julian A. Martinez-Agosto, MD ${ }^{15,16}$, Rüdiger Lorenz, MD ${ }^{17}$, \\ Naama Orenstein, MD ${ }^{18,19}$, Jonathan N. Berg, MD ${ }^{20,21}$, Jeanne Amiel, MD ${ }^{22}$, Delphine Heron, MD $^{23}$, \\ Boris Keren, MD, PhD ${ }^{23}$, Jan-Maarten Cobben, $\mathrm{MD}^{24,25}$, Leonie A. Menke, MD, PhD ${ }^{24}$, \\ Elysa J. Marco, MD ${ }^{26}$, John M. Graham Jr, MD, ScD ${ }^{27}$, Tyler Mark Pierson, MD, PhD ${ }^{28}$, \\ Ehsan Ghayoor Karimiani, MD, PhD ${ }^{29}$, Reza Maroofian, PhD ${ }^{29}$, M. Chiara Manzini, PhD ${ }^{30}$, \\ Edmund S. Cauley, $\mathrm{MSC}^{30}$, Roberto Colombo, MD ${ }^{31,32}$, Sylvie Odent, $\mathrm{MD}^{33}$, \\ Christele Dubourg, PharmD, PhD ${ }^{34}$, Chanika Phornphutkul, MD ${ }^{35}$, Arjan P. M. de Brouwer, PhD ${ }^{1}$, \\ Bert B. A. de Vries, MD, $\mathrm{PhD}^{1}$ and Anneke T. Vulto-vanSilfhout, $\mathrm{MD}, \mathrm{PhD}^{1}$
}

Purpose: To investigate the effect of different DEAF1 variants on the phenotype of patients with autosomal dominant and recessive inheritance patterns and on DEAF1 activity in vitro.

Methods: We assembled a cohort of 23 patients with de novo and biallelic DEAF1 variants, described the genotype-phenotype correlation, and investigated the differential effect of de novo and recessive variants on transcription assays using DEAF1 and Eif4g3 promoter luciferase constructs.

Results: The proportion of the most prevalent phenotypic features, including intellectual disability, speech delay, motor delay, autism, sleep disturbances, and a high pain threshold, were not significantly different in patients with biallelic and pathogenic de novo DEAF1 variants. However, microcephaly was exclusively observed in patients with recessive variants $(p<0.0001)$.

Conclusion: We propose that different variants in the DEAF1 gene result in a phenotypic spectrum centered around neurodevelopmental delay. While a pathogenic de novo dominant variant would also incapacitate the product of the wild-type allele and result in a dominant-negative effect, a combination of two recessive variants would result in a partial loss of function. Because the clinical picture can be nonspecific, detailed phenotype information, segregation, and functional analysis are fundamental to determine the pathogenicity of novel variants and to improve the care of these patients.

Genetics in Medicine (2019) 21:2059-2069; https://doi.org/10.1038/s41436019-0473-6

Keywords: DEAF1; neurodevelopmental disorder; intellectual disability; genotype; phenotype

\section{INTRODUCTION}

DEAF1 (MIM *602635; NM_021008.3) encodes the deformed epidermal autoregulatory factor-1 homolog (DEAF1), a transcription factor that is highly expressed in the central nervous system, in particular during early embryogenesis. ${ }^{1}$ DEAF1 regulates the expression of various genes ${ }^{2}$ as both a transcriptional activator and repressor. ${ }^{3-6}$ It contains several functional domains including a centrally located SAND (Sp100, AIRE, NucP41/75, and DEAF1) domain, a zinc finger motif (ZnF), a nuclear localization signal (NLS), a nuclear export signal (NES), and an MYND (myeloid translocation protein 8 , Nervy, and DEAF1) domain (Fig. 1) $\left(\right.$ refs. $^{6,7}$ ). The region encompassing the SAND domain constitutes a DNAbinding domain, which binds to TTCG motifs. ${ }^{6}$ The SAND and MYND domains are also involved in protein-protein interactions. ${ }^{6-9}$ The presence of a NLS and NES indicates that DEAF-1 may be regulated by nuclear/cytoplasmic shuttling. ${ }^{7}$ Biallelic disruption of Deaf1 in mice results in neural tube defects, ${ }^{10}$ and biallelic Drosophila Deaf1 loss-of-function mutants show early embryonic arrest. ${ }^{11}$ 
a DEAF1 pre-mRNA 1

708

$\begin{array}{llllllll}997 & 1095 & 1225 & 1372 & 15121578 & 1705 & 1834 & 1963\end{array}$

221123012405

735

b DEAF1 protein

$5^{\prime}$ M 2 M M 4 5 M 6 M M 8 M 9 M 10 M11M 12

c

$$
\text { N-terminus }
$$

$198 \quad 272 \quad 30+306 \quad 26$

$453,476<04540565$
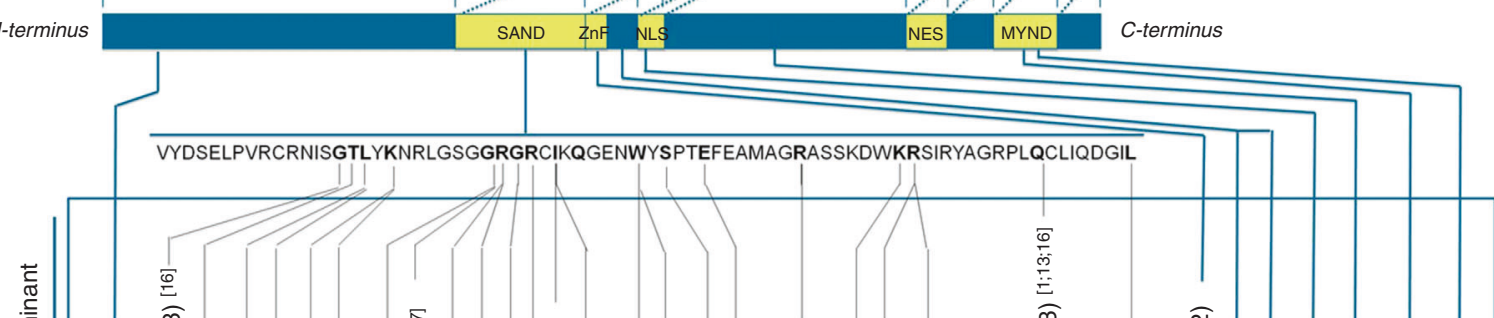

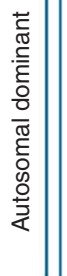

E

II

c.

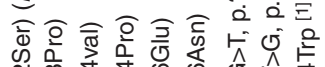

N

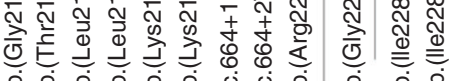

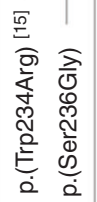

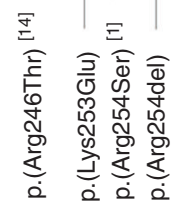

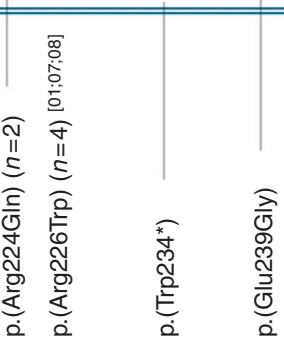

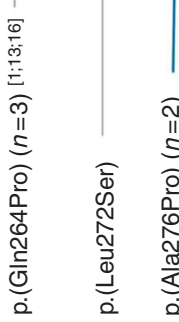

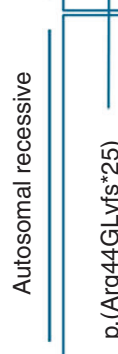

(a)

e

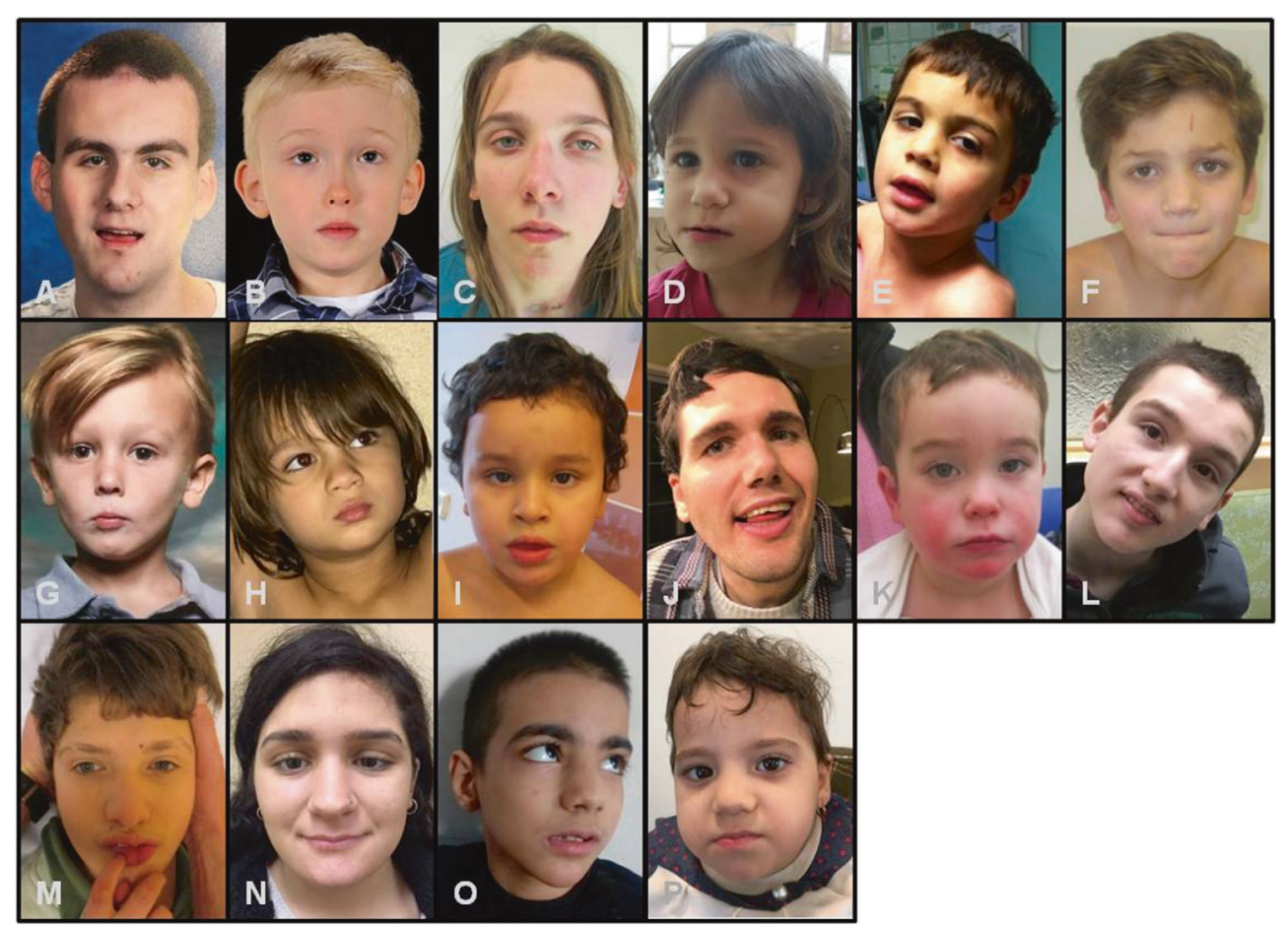

Pathogenic variants in the DEAF1 gene have been reported to lead to two clinically distinct intellectual disability (ID) syndromes: autosomal dominant mental retardation 24 (MRD24; MIM 615828) caused by de novo variants, ${ }^{1,12-17}$ and the recessively inherited dyskinesia, seizures, and intellectual developmental disorder syndrome (DYSEIDD; MIM 617171) (refs. ${ }^{16,18-20}$ ). These two syndromes are collectively described as DEAF1-associated neurodevelopmental disorders (DAND). To date, nine different de novo pathogenic variants have been described in ten individuals, 
Fig. 1 Genotype and facial phenotype of individuals with DEAF1-associated neurodevelopmental disorders (DAND). a Structure of the DEAF1 precursor messenger RNA (pre-mRNA) [NM_021008.3] with light blue boxes indicating the 5' and 3' untranslated regions (UTRs) and dark blue boxes representing the coding region of exons 1 to 12, connected by lines indicating introns (not drawn to scale). b Structure of DEAF1 protein [NP_066288], including the DNA-binding and dimerization SAND domain, the zinc finger homology domain (ZnF) also involved in DNA-binding, nuclear localization signal (NLS), nuclear export signal (NES), and MYND domain. Distribution of (c) de novo heterozygous variants leading to autosomal dominant mental retardation 24 and (d) homozygous or compound heterozygous variants leading to autosomal recessive dyskinesia, seizures, and intellectual developmental disorder. The number of patients described with the depicted variant are indicated between parentheses if the variant was indentified in more than one case. Novel variants are indicated in bold. e Nonspecific facial dysmorphisms of individuals with pathogenic de novo DEAF1 variants (A-L) and biallelic DEAF1 variants $(\mathbf{M}-\mathbf{P})$, showing common characteristics as horizontal eyebrow, broad nasal tip, exaggerated Cupid's bow, thick lower lip vermilion, and pointed chin: (A) individual with p.(Gly212Ser), at 20 years old (AD/1); (B) individual with p.(Thr213Pro), at 7 years old (AD/3); (C) individual p.(Leu214Val), at 14 years old (AD/ 4); (D) individual with p.(Leu214Pro), at 3 years old (AD/5); (E) individual with p.(Lys216Glu), at 10 years old (AD/6); (F) individual with p.(Lys216Asn), at 7 years old (AD/7); (G) individual with p.(Pro174_Gly222del), at 6 years old (AD/8); (H) individual with p.(Gly225Glu), at 5 years old (AD/9); (I) individual with p. (Ser236Gly), at 4 years old (AD/11); (J) individual with p.(Arg254del), at 25 years old (AD/13); (K) individual with p.(Leu272Ser), at 2 years old (AD/15); (L) individual with p.(Ala276Pro), at 14 years old (AD/17); (M) individual with p.(Asp369Alafs*51) and p.(Cys540Metfs*18), at 17 years old (AR/1); (N) individual with p.(Trp234*) and p.(Glu239Gly), at 16 years old (AR/3); (O) individual with p.(Arg224Gln) in homozygosity, at 10 years old (AR/4); (P) individual with $p$. (Arg224Gln) in homozygosity), at 2 years old (AR/5). Individuals with de novo variants may have high forehead and/or macrocephaly, while individuals with biallelic variants may have narrow forehead and microcephaly.

who manifested moderate to severe ID with severely affected expressive speech and mild motor delay. ${ }^{1,12-17}$ Epilepsy was described in half of the individuals. ${ }^{16}$ Behavioral problems consisted of hyperactive, compulsive, and/or aggressive behavior; fascination with water; and striking mood swings. ${ }^{114-16}$ Other characteristics were autism, recurrent infections, a high pain threshold, and an abnormal walking pattern. ${ }^{1,14-16}$ Body measurements were normal, facial dysmorphisms were only mild, and no other major congenital anomalies were observed. ${ }^{1}$ All but one of the de novo pathogenic DEAF1 variants occurred in the SAND domain, including seven missense variants and one splice-site variant. $^{1,12-17}$ One in-frame deletion of three base pairs was reported in the NLS domain. ${ }^{16}$ These de novo pathogenic $D E A F 1$ variants impair the DEAF1 transcriptional activity, DNA-binding, and/or alter subcellular localization.,16 Because heterozygous DEAF1 deletions ${ }^{21}$ do not cause DAND, de novo pathogenic variants have been proposed to lead to a dominant-negative effect. ${ }^{1}$

Two kindreds with patients with homozygous DYSEIDD pathogenic DEAF1 variants were previously reported: a missense change and a noncanonical splice-site pathogenic variant. $^{16,18-20}$ In total, seven patients homozygous for either of these variants presenting with ID, microcephaly, and hypotonia, were described. ${ }^{16,18-20}$ Seizures and white matter abnormalities resulting in dyskinesia were reported in four of the six patients for whom these features were investigated. ${ }^{18-20}$ The missense variant was present in the SAND domain as well, whereas the splice-site variant resulted in exon skipping and reduced the normal fulllength messenger RNA (mRNA) copy number in the patients to $5 \%$ of the wild-type (WT) level, ${ }^{19}$ suggesting that the recessive phenotype resulted from a partial loss of function of DEAF1.

To investigate the effect of different DEAF1 variants on the phenotype of patients with autosomal dominant and recessive inheritance patterns, we have assembled a cohort of 23 patients not previously reported with de novo and biallelic DEAF1 variants. We describe the genotype-phenotype correlation and investigate the differential effect of de novo and recessive variants on DEAF1 activity in vitro.

\section{MATERIALS AND METHODS \\ Identification of individuals}

Patients were referred by physicians working in intellectual disability support services and by clinical geneticists. Written informed consent was obtained for all individuals involved, including consent to publish patient photos. This study adhered to the World Health Association Declaration of Helsinki (2013) and was approved by the institutional review board Commissie Mensgebonden Onderzoek Regio ArnhemNijmegen. Genomic DNA was isolated from peripheral blood samples following standard procedures. ${ }^{22}$ Exome enrichment, high-throughput sequencing, and subsequent de novo analysis was performed by established procedures. ${ }^{23}$ Copy-number variant analysis was performed on exome data by using CoNIFER $^{1,24}$ as described previously. ${ }^{25}$

\section{Plasmid constructs}

DEAF1 mammalian expression and DEAF1 and Eif4g3 promoter luciferase plasmids have been previously described. ${ }^{1}$ Site-directed mutagenesis was used to introduce the specific amino acid substitutions into the DEAF1 expression plasmids, derived from the human DEAF1 complementary DNA (cDNA) (GenBank accession number AF049459). Variants resulting in the indicated human amino acid substitutions were generated by polymerase chain reaction (PCR) as previously described. ${ }^{1}$

\section{Transcription assays}

Luciferase assays using DEAF1 and Eif4g3 promoter luciferase constructs have been previously described. ${ }^{1}$ Briefly, HEK293T cells in 24-well plates were transfected with 125 ng pcDNA3 (control) or DEAF1 (WT or DEAF1 variants) expression plasmids with $375 \mathrm{ng}$ promoter luciferase and 1.25 ng RSV-Renilla luciferase constructs using the calcium phosphate technique for 18 hours. Luciferase assays were 
performed 24 hours later using the Dual-Luciferase Reporter Assay System (Promega, Madison, WI, USA).

\section{DEAF1 EBV-LCL RNA analysis}

Epstein-Barr virus-transformed lymphoblastoid cell line (EBV-LCL) cells $\left(8 \times 10^{6}\right.$ cells) were grown in $20 \mathrm{~mL}$ Roswell Park Memorial Institute medium (RPMI) supplemented with $15 \%$ fetal bovine serum, penicillin, and streptomycin; 24 hours later, $10 \mathrm{~mL}$ was removed to a new flask and cells were treated with vehicle (water) or cycloheximide $(50 \mathrm{ug} / \mathrm{mL})$ for 6 hours. RNA was isolated from EBV-LCL cells using Trizol reagent and $1.0 \mathrm{ug}$ of RNA was reverse transcribed. PCR was performed using cDNA and primers to amplify specific regions of DEAF1 cDNA for downstream restriction digests or subcloning and DNA sequencing. Quantitative PCR was performed using DEAF1 (1617dup) and GAPDH primers ${ }^{26}$ and data were normalized using the $2^{(-\Delta \Delta \mathrm{Ct})}$ method. ${ }^{27}$

\section{Immunoprecipitation and western blot}

EBV-LCLs were lysed in lysis buffer containing $150 \mathrm{mM}$ sodium chloride, $50 \mathrm{mM}$ Tris $(\mathrm{pH}=7.5), 1.0 \%$ TritonX-100, $1.0 \mathrm{mM}$ EDTA, $1.0 \mathrm{mM}$ sodium fluoride, $0.2 \mathrm{mM}$ sodium orthovanadate, aprotinin $(10 \mu \mathrm{g} / \mathrm{mL})$, leupeptin $(10 \mu \mathrm{g} / \mathrm{mL})$, and pepstatin $(10 \mu \mathrm{g} / \mathrm{mL})$ and lysates were incubated with rabbit DEAF1 antibodies ${ }^{8}$ or preimmune serum bound to protein $\mathrm{G}$ magnetic beads overnight at $4{ }^{\circ} \mathrm{C}$. Beads were washed and proteins were eluted in Laemmli sample buffer. Transfected HEK293t cells were lysed in lysis buffer. Proteins were separated on sodium dodecyl sulfate polyacrylamide gel electrophoresis (SDS-PAGE) gels and transferred to polyvinylidene fluoride membranes. Immunoblot analysis was performed with a different rabbit anti-DEAF1 antibody for EBV-LCL immunoprecipitation experiments or rabbit antiDEAF1 and mouse anti-bACTIN (Abcam, Cambridge, MA, USA) for transfected HEK293t experiments.

\section{Genotype-phenotype correlations}

Fisher's exact test, using a two-sided $p$ value, was carried out to compare proportions of clinical features between groups of patients with de novo and biallelic DEAF1 variants. After conducting Bonferroni correction, a $p$ value of $<0.0025$ was considered significant.

\section{RESULTS}

\section{De novo variants in the SAND domain of DEAF1 impair transcriptional activity}

We identified 14 novel and two previously published de novo $D E A F 1$ variants in a total of 18 patients by exome sequencing $(n=16)$, genome sequencing $(n=1)$, and autism gene panel analysis $(n=1)$ performed in 17 medical centers from all over the world (Fig. 1; Table S1). These variants included 14 missense variants, one in-frame deletion that resulted in a single amino acid change, and one splice-site variant, c.664 $+1 \mathrm{G}>\mathrm{T}$. To determine if the $\mathrm{c} .664+1 \mathrm{G}>\mathrm{T}$ variant resulted in alternatively spliced DEAF1 mRNA transcripts, PCR was used to amplify regions containing exons 2-5 using EBV-LCL
mRNA from individual AD/8. DNA sequencing indicated the variant causes skipping of exon 4 , which results in an in-frame deletion of 49 amino acid residues in the SAND domain p. (Pro174_Gly222del) (Fig. 2). Compared with control EBVLCL, a truncated DEAF1 protein, as well as full-length DEAF1, were observed in patient AD/8 EBV-LCL lysate and corresponded to a similar molecular weight protein using lysates from cells transfected with WT or p.(Pro174_Gly222del) plasmid DNA. The novel variant p.(Ala276Pro) was found in two unrelated individuals from this study. The previously reported de novo variants p.(Gly212Ser) and p. (Gln264Pro) $)^{1,13,16}$ were identified in our cohort in two and one families, respectively. The p.(Gly212Ser) variant was identified in 2 of 247 reads in peripheral blood cells of the patient's unaffected father. The variant was not detected by Sanger sequencing, suggesting that he is likely a mosaic for this variant.

Fifteen of the 16 de novo variants are located in the SAND or adjacent $\mathrm{ZnF}$ domain; the p.(Pro293Leu) variant is between the $\mathrm{ZnF}$ and NLS (Fig. 1c). All missense variants were predicted by SIFT $^{28}$ and PolyPhen-2 (ref. ${ }^{29}$ ) to be diseasecausing and had a CADD score $>20.0$ (ref. ${ }^{30}$ ). None of the novel de novo variants were found in gnomAD. ${ }^{31}$ Multispecies alignment showed that all mutated amino acids are highly conserved across species (Figure S1).

DEAF1 binds and represses its own promoter activity. ${ }^{32}$ $D E A F 1$ promoter transcriptional repression activity was analyzed for de novo variants. All variants lost transcriptional repression activity compared with wild-type DEAF1, although p.(Pro293Leu) retained some repression activity (Fig. 3a). DEAF1 also increases the transcriptional activity of the mouse Eif4g3 promoter $^{5}$ and de novo DEAF1 variants have previously been shown to suppress activation of this promoter. $^{1}$ In 12 of our 14 variants, an approximate tenfold suppression of transcriptional activity relative to basal expression levels was demonstrated. No activation or reduction in promoter activity was observed for p.(Ser236Gly) relative to basal activity, and p.(Pro293Leu) increased promoter activity (Fig. 3 b).

\section{Biallelic variants in DEAF1 result in no significant effect in transcriptional assays}

Six novel biallelic DEAF1 variants were found in compound heterozygosity in three unrelated individuals and one novel homozygous variant was identified in two siblings of a consanguineous family by using exome sequencing (Fig. 1d; Table S2). These variants were scattered throughout DEAF1. In two families, compound heterozygous variants, p. (Trp234*) and p.(Glu239Gly), or a homozygous variant, p. (Arg224Gln), were found in the SAND domain. The compound heterozygous missense variant p.(Glu239Gly) is not reported in gnom $A D$ and is predicted by SIFT to be deleterious, by PolyPhen-2 to be probably damaging, and had a CADD score 25. The homozygous substitution p. (Arg224Gln) located in the SAND domain is present only once heterozygously in gnomAD (frequency of $4.065 \mathrm{e}-6$ ), was 
a

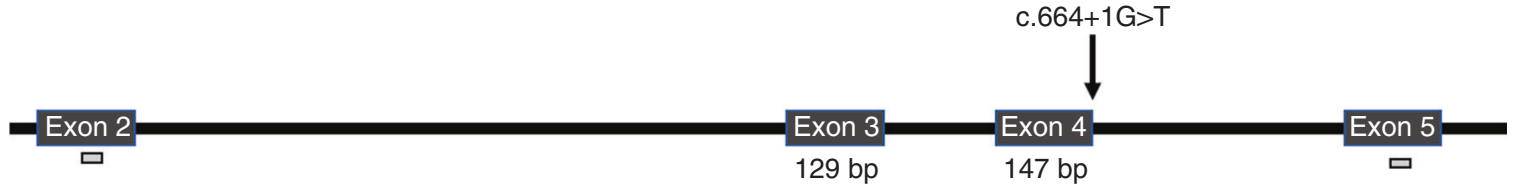

b

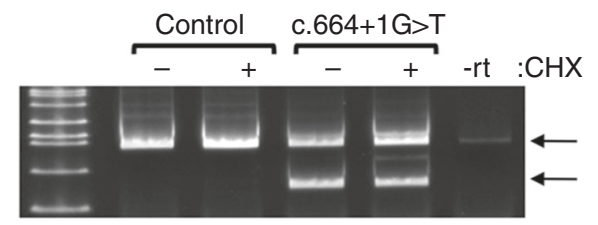

d

e
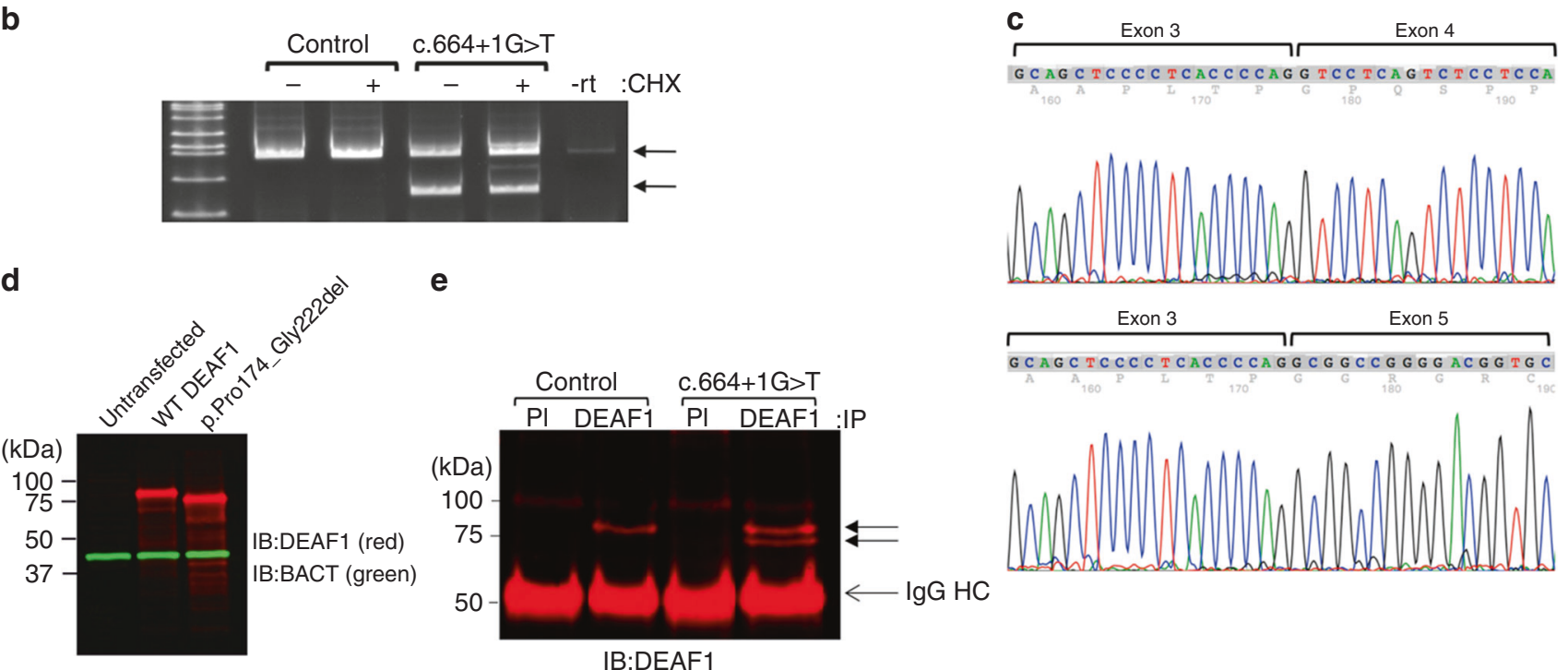

Fig. 2 The de novo variant c.664 $+\mathbf{1 G}>$ T results in an in-frame deletion within the DEAF1 SAND domain. a Location of the c.664+1G>T variant relative to exons $2-5$ in the DEAF1 gene. b Polymerase chain reaction (PCR) using primers that would amplify exons 2-5 of DEAF1 messenger RNA (mRNA) (gray bars shown in a) with complementary DNA (CDNA) from control or c.664+1G>T Epstein-Barr virus-transformed lymphoblastoid cell line (EBV-LCL) (individual $\mathrm{AD} / 8$ ) treated with vehicle $(-)$ or cycloheximide $(\mathrm{CHX})(+)$. A single DNA band was observed in control EBV-LCL corresponding to correctly spliced DEAF1. An additional, approximately 150-bp smaller PCR product was identified in c.664+1G> T EBV-LCL. c DNA sequencing indicated c.664+1G>T EBVLCL has both correctly spliced DEAF1 RNA (above) as well as DEAF1 mRNA that lacks exon 4 (below), which could result in a truncated p.Pro174_Gly222del DEAF1 protein. $\mathbf{d}$ Lysates from HEK293t cells transfected with the expression constructs for wild-type (WT) ( 80 kDa) or p.(Pro174_Gly222del) variant ( 75 $\mathrm{kDa}$ ) were separated by sodium dodecyl sulfate polyacrylamide gel electrophoresis (SDS-PAGE) and analyzed by western blot using DEAF1 (red) and b-ACTIN (green) antibodies. e Lysates from control or c.664+1G> T EBV-LCL were immunoprecipitated with preimmune serum (PI) or DEAF1 antibodies followed by western blots with DEAF1 antibodies. The control EBV-LCL shows a single 80-kDa full-length DEAF1 protein band. An additional 75 kDa DEAF1 protein was also observed in the EBV-LCL with the c.664+1G>T variant. Closed arrows indicate DEAF1 protein bands and open arrow indicates heavy chain $\lg G(\lg G \mathrm{HC})$.

predicted by SIFT to be tolerated, by PolyPhen-2 to be disease-causing, and had a CADD score 31 . In the other two families, a single base-pair duplication or deletion resulting in frameshift near to the $5^{\prime}$ of DEAF1, p.(Arg44Glyfs $\left.{ }^{\star} 25\right)$, or in the middle of the DEAF1 gene, p.(Asp369Alafs $\left.{ }^{\star} 51\right)$, both located outside a domain, occurred in combination with a variant in the MYND domain, respectively an in-frame deletion, p.(Phe527del) or an insertion resulting in a frameshift, p.(Cys540Metfs $\left.{ }^{\star} 18\right)$. Three of these variants, p.(Arg44Glyfs $\left.{ }^{\star} 25\right)$, p. $\left(\operatorname{Trp} 234^{\star}\right)$, and p.(Asp369Alafs $\left.{ }^{\star} 51\right)$, are expected to lead to nonsense-mediated decay (NMD) of their respective transcripts.

DEAF1 mRNA expression analysis was performed using EBV-LCL from patient AR/1, who carried c.1617dup, p. (Cys540Metfs $\left.{ }^{\star} 18\right)$ and c.1104_1105dup, p.(Asp369Alafs ${ }^{\star} 51$ ) in compound heterozygosity, and from parents carrying either the c.1617dup or c.1104_1105dup. To differentiate the expression of DEAF1 transcripts from each allele, two different PCR amplicons were generated that contain either c.1617dup or c.1104_1105dup variant from patient and parent EBV-LCL treated with vehicle or cycloheximide (CHX), an inhibitor of NMD. The c.1617dup eliminates an endogenous NdeI site and the c.1104_1105dup generates a new HhaI site. Restriction endonuclease digestion analysis indicated that both c.1617dup or c.1104_1105dup containing mRNA transcripts were present in patient and parent cDNA. The c.1104_1105dup transcript seemed to be present in a lower amount in cells not treated with $\mathrm{CHX}$ as compared with those treated with CHX. As CHX is an inhibitor of NMD, this suggests that this transcript is subject to NMD (Figure S2). DEAF1 immunoprecipitations were performed on EBV-LCL lysates from patient and parents. Compared with parent EBVLCL, no full-length or p.(Cys540Metfs $\left.{ }^{\star} 18\right) \sim 80-\mathrm{kDa}$ DEAF1 protein was observed in patient lysate. A faint truncated $\sim 60$ $\mathrm{kDa}$ band consistent with the p.(Asp369Alafs^51) DEAF1 protein was observed in patient and parent c.1104_1105dup. Quantitative PCR (qPCR) showed that DEAF1 mRNA expression in untreated EBV-LCL of the patient AR/1 (c.1104_1105dup/c.1617dup variants) was 30\% of normal EBV-LCL expression levels $(p<0.05)$ (Figure S3). CHX 

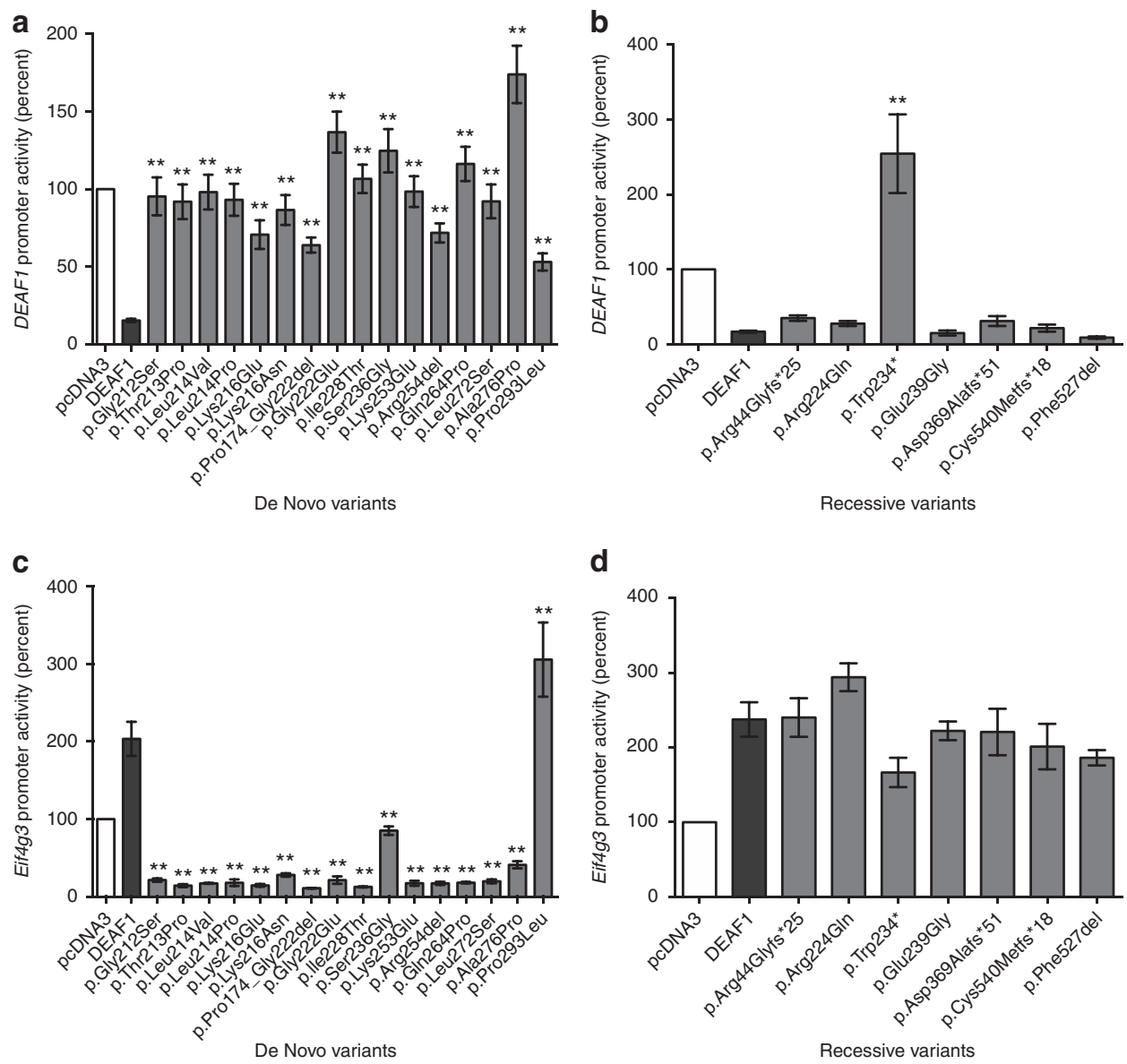

Fig. 3 Effect of DEAF1 variants on transcriptional activity. a, b DEAF1 promoter activity after transfection of HEK293t cells with wild-type (WT) or the indicated variants. a Compared with WT, all de novo variants resulted in loss of transcriptional repression activity at the DEAF1 promoter. b Heritable biallelic variants had no effect on DEAF1 promoter transcriptional repression, except for $\mathrm{p}$.(Trp234*). c, d Eif4g3 promoter activity after transfection of HEK293t cells with WT or the indicated variants. (c) All de novo variants, except p.(Pro293Leu) and p.(Ser236Gly), resulted in suppression of transcription relative to WT transactivation. No change from basal promoter activity (pcDNA3) was detected for $p$.(Ser236Gly) and increased promoter activity was observed for $p$. (Pro293Leu). d Heritable biallelic variants had no effect on Eif4g3 promoter activation compared with WT. Each bar represents the mean $+/-$ SEM of the normalized luciferase activity of three independent experiments when the activity of pcDNA3 (DEAF1 promoter alone) was set to $100 \%(N=5-7$ de novo variants with $N=14-15$ WT, $N=4-5$ heritable variants with $N=10-12$ WT). ${ }^{*} p<0.01$ One-way analysis of variance (ANOVA) with Dunnett's multiple comparison WT DEAF1 versus each mutant.

treatment significantly increased DEAF1 mRNA expression relative to vehicle-treated cells in the c.1104_1105dup/ c.1617dup patient as well as the parent carrying the c.1104_1105dup supporting that the c.1104_1105dup-containing transcript is targeted by NMD.

The effects of the biallelic variants on DEAF1 transcriptional activity, protein expression, and cellular localization were also determined. Compared with WT DEAF1, no change in transcriptional repression activity was observed for the biallelic variants, except for p.(Trp234*) (Fig. 3c). No significant change in transcriptional activation of the Eif $4 g 3$ promoter was observed (Fig. 3d). Interestingly, p.(Arg44Glyfs $\left.{ }^{\star} 25\right)$, which should result in a severely truncated DEAF1 protein that lacks most of the DEAF1 protein domains including the SAND domain, retained transcriptional activity. Western blots were performed using lysates from HEK293t transfected with WT or DEAF1 biallelic variants. A faint $\sim 70-\mathrm{kDa}$ protein was observed for $\mathrm{p}$. (Arg44Glyfs ${ }^{\star} 25$ ) (Figure S4). This might be the result of the use of a translational start at a downstream in-frame AUG start codon located at codon 69 . The p.(Arg44Glyfs $\left.{ }^{\star} 25\right)$ variant results in a stop codon $5^{\prime}$ adjacent to methionine 69 of DEAF1. The p. $\left(\operatorname{Trp} 234^{\star}\right)$ and p.(Asp369Alafs $\left.{ }^{\star} 51\right)$ variants resulted in truncated DEAF1 proteins approximately 30 and $60 \mathrm{kDa}$ in size, respectively. The p. (Cys540Metfs $\left.{ }^{\star} 18\right)$ variant resulted in a slightly lower molecular weight protein. As expected p.(Arg224Gln), p.(Glu239Gly), and p.(Phe527del) variants had the same apparent molecular weight as WT DEAF1. Immunofluorescent staining was used to determine the subcellular localization of the biallelic DEAF1 variants in transfected cells. Unlike WT DEAF1, which localized to the nucleus, p.(Trp234*) was found throughout the cell (Figure S5). The other biallelic variants localized to the nucleus. 
De novo DEAF1 variants result in a nonspecific phenotype comprising ID, speech delay, motor delay, sleep disturbance, autism, and a high pain threshold

Fourteen male and three female individuals with a de novo $D E A F 1$ variant (median age: 7.5 years; age range: $2.5-38$ years), were clinically characterized (Table 1; Table S3). All were born from healthy unrelated nonconsanguineous families. This cohort consisted of 11 children, 6 of whom were preschool children, 3 were adolescents, and 3 were adults. One individual was excluded from genotype-phenotype analysis because he had the variant $\mathrm{p}$. (Pro293Leu), which was not located in the SAND domain and did not result in suppression, but rather in an increase of Eif4g3 promoter activity.

Developmental delay (DD)/ID was present in all patients $(17 / 17 ; 100 \%)$ and was typically moderate-severe or severe DD/ID (Table S4). Motor delay, present in most of the patients $(12 / 17 ; 71 \%)$, was usually mild. Language development was severely delayed in all patients, except for one patient with the variant p.(Leu214Val) (16/17; 94\%). Seven patients had absent speech and nine had limited speech. Of note, nonverbal patients were said to have used a few words before losing the ability to produce speech. Developmental regression was described in half of the individuals, mostly corresponding to loss of the ability to communicate verbally. Although in most individuals expressive language was severely impaired, receptive language was perceived, particularly by caregivers, as better than expressive. Furthermore, patients $(15 / 15 ; 100 \%)$ were able to communicate simple needs nonverbally, for instance taking the caregivers' hand to point or lead to the item(s) of interest, using gestures and sign language, producing sounds accompanied (or not) by gestures (e.g., to express emotions, as annoyance), or using a picture exchange communication system, picture prompts, a tablet, or a touch-based speech-generating device.

Autism spectrum disorder occurred in all patients $(16 / 16$; $100 \%)$. In the majority, poor eye contact $(12 / 16 ; 75 \%)$; fascinations $(15 / 17 ; 88 \%)$, particularly a compulsive interest in water; and sudden mood swings (16/17; 94\%) were concomitantly observed. Mood swings were characterized by exaggeration of emotional affects, with paroxysmal bursts of laughter and context-inappropriate happy disposition alternating with aggressive behavior or depressive feelings. Aggressive behavior (12/16; 75\%) manifested both as heteroaggression and autoaggression (e.g., self-mutilation, head-banging, self-biting). Other behavioral problems $(13 / 15 ; 87 \%)$ included pica, impulsivity/hyperactive behavior, and obsessive traits.

Epilepsy was also observed in the majority of patients $(10 / 14 ; 71 \%)$. Median age at diagnosis was 3 years, although age of onset varied from 2 days old to 16 years old (Table S5). Seizures were usually generalized and frequentlydifficult to treat or intractable $(9 / 10 ; 90 \%)$. Antiepileptic drugs (AEDs) were able to reduce seizures in five patients, while in four other patients seizures were refractory to treatment. Of note, seizures were noticed to adversely influence speech.

Sleep dysfunction was frequent and severe (15/17; 88\%). It consisted of difficulty falling asleep (sleep onset insomnia) and awaking frequently (maintenance insomnia). Patients were reported to be very disruptive when waking during the night. In some patients, sleeping problems were treated with some benefit using medication, such as melatonin.

Other neurologic abnormalities included a remarkably high pain threshold (14/16;88\%), hypotonia (10/16;63\%), and gait difficulties, comprising of gait ataxia $(4 / 16 ; 25 \%)$ or an ataxic looking gait $(12 / 17 ; 71 \%)$, as broad-based and imbalanced gait, or a tip-toe gait. Brain abnormalities as indicated by brain magnetic resonance image (MRI) were present in less than a third of the patients $(4 / 13 ; 31 \%)$, but no common pattern of brain malformations could be distinguished. Movement disorder, in particular dystonia, was only reported in one individual.

Non-neurodevelopmental issues included recurrent infections that occurred in more than half of patients $(10 / 16 ; 63 \%)$, mostly in infancy or childhood. They included predominantly chronic ear infections, sinusitis, or upper respiratory tract infections. In addition, gastrointestinal abnormalities were common (12/16; 75\%) and included feeding difficulties, gastroesophageal reflux disease, constipation, and diarrhea. Craniofacial dysmorphisms were mild (Fig. 1e) and macrocephaly was observed in approximately one quarter of individuals $(4 / 17 ; 24 \%)$. Growth was usually within the norm for age and gender.

\section{Biallelic DEAF1 variants result in microcephaly}

Microcephaly $(3 / 5 ; 60 \%)$ was exclusively observed in patients with recessive variants. All patients with biallelic DEAF1 variants manifested ID and developmental regression, except for one (Tables S6 and S7). All parents were healthy. Remarkably, although the patient with variants p.(Asp369Alafs $\left.{ }^{\star} 51\right)$ and p.(Cys540Metfs $\left.{ }^{\star} 18\right)$ in compound heterozygosity never learned to walk and had absent speech, similarly to other patients with biallelic DEAF1 variants, he completed various exams from the first grade of secondary school, suggesting he has a normal cognitive function, although this was not formally tested. Additionally, he used nonverbal communication aids (supportive typing) that revealed good receptive language. Behavioral problems occurred in all patients and ranged from poor eye contact $(4 / 5 ; 80 \%)$ to autism $(1 / 4 ; 25 \%)$ and aggressive behavior $(2 / 5 ; 40 \%)$. Frequent neurologic abnormalities included epilepsy (4/5; $80 \%$ ) (Tables S5), sleep disturbance $(5 / 5 ; 100 \%)$, and brain abnormalities $(4 / 5 ; 80 \%)$, such as ventriculomegaly with or without hydrocephalus, corpus callosum hypoplasia, or white matter abnormalities. Movement disorder $(2 / 4 ; 50 \%)$ included dystonia and stereotypies. Common extraneurologic features included feeding difficulties $(3 / 5 ; 60 \%)$ and constipation $(5 / 5$; $100 \%)$. Lastly, facial dysmorphisms were minor and nonspecific (Fig. 1e). 
$\frac{\breve{Q}}{\frac{\pi}{2}}$
Q

$\widehat{\simeq}$

II

बृँ

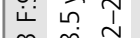

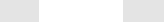

$\pi$

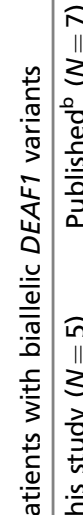

这

㟧

$\stackrel{Ð}{\rightleftarrows}$

$\stackrel{ \pm}{ \pm}$

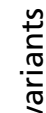

誌

$\frac{\frac{u}{0}}{\frac{\sqrt{0}}{a}}$

$\frac{\pi}{0}$

व

$$
\text { 政 }
$$

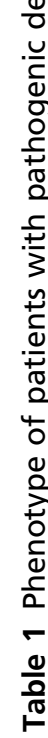

$\frac{m}{m} \delta \Sigma \delta \frac{m}{m} \stackrel{m}{m} \frac{\Sigma}{z} \equiv$

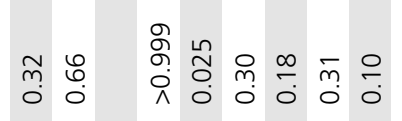

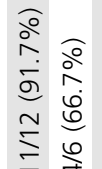

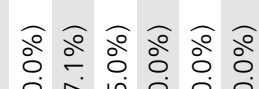

क n

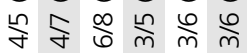

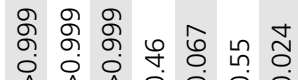

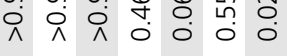

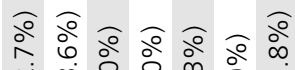
i

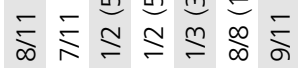

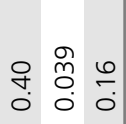

○े

$\Sigma \stackrel{m}{=} \stackrel{m}{m}$

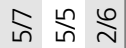

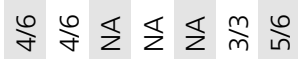

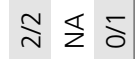

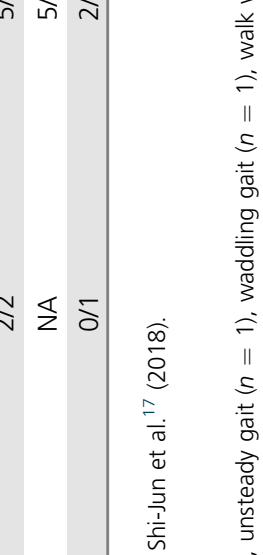

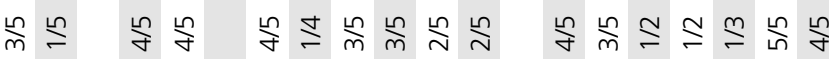

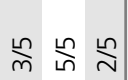

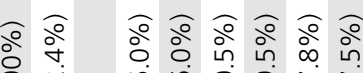

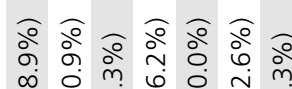

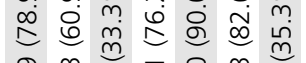

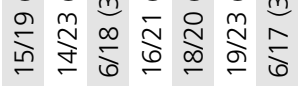

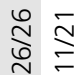

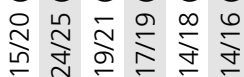

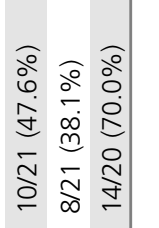

회 


\section{De novo and biallelic variants result in a similar phenotype except for microcephaly}

The proportion of the most prevalent phenotypic features, including ID, speech delay, motor delay, autism, sleep disturbances, and a high pain threshold, was not significantly different in patients with biallelic and pathogenic de novo $D E A F 1$ variants, when considering all patients included in this study and previously described individuals (Table 1). Interfamilial phenotypic variability was observed among patients with the same de novo variant (e.g., some patients developed seizures, while others did not). Microcephaly, however, was observed exclusively in patients with recessive variants $(p<$ $0.0001)$.

\section{DISCUSSION}

In this study, we expanded the clinical consequences of variants implicated in DEAF1-associated neurodevelopmental disorders (DAND) by describing the phenotype of 17 patients with a de novo pathogenic variant and 5 patients with biallelic variants in the DEAF1 gene. We determined that the de novo variants impaired DEAF1 transcriptional repression activity and 15 of the 17 variants reversed DEAF1-mediated transcriptional activation at the Eif $4 g 3$ promoter. Apart from the $\mathrm{p}\left(\operatorname{Trp} 234^{*}\right)$ variant, the other six novel recessive DEAF1 variants demonstrated no change in transcriptional activity.

We identified missense variants in the SAND domain, both de novo and recessive. Only de novo variants, however, altered $D E A F 1$ and Eif4g3 promoter activity. This is in line with previous data implying a dominant-negative effect of these variants. ${ }^{1}$ So far, no de novo nonsense or frameshift variants resulting in disease have been identified. De novo variants clustered in very close proximity within the SAND domain. Moreover, variants have been found recurrently affecting the same residues, namely p.Gly212, p.Leu214, p.Lys216, p.Ile228, p.Arg254, p.Gln264, and p.Ala276 (refs. ${ }^{1,12-20}$ ). Additionally, the canonical donor splice site $3^{\prime}$ of c.664 was also found to be repeatedly affected. ${ }^{17}$ We show in our study that c.664+1G>T causes skipping of exon 4, producing an in-frame deletion in the SAND domain that results in abnormal transcriptional activity mimicking the effect of other missense variants on DEAF1 protein activity.

Missense biallelic variants in the SAND domain do not impair the function of this domain as evidenced by the normal transcriptional activity of the mutant protein in our assays. This is especially remarkable for the novel homozygous variant p.(Arg224Gln), as the previously reported de novo p.(Arg224Trp) variant was shown to result in loss of transcriptional repression of the DEAF1 promoter, and was unable to activate the Eif $4 g 3$ promoter. ${ }^{1}$ We hypothesize that the p.(Arg224Gln) variant affects normal protein folding of DEAF1 because arginine, an amino acid with an electrically charged side chain, is replaced by an amino acid with a polar uncharged chain. This is also the case for the recessive $\mathrm{p}$. (Arg226Trp) variant, also located in the SAND domain. ${ }^{16,18,20}$ Similarly, the compound heterozygous variant p.(Glu239Gly), which results in a protein with an apparently normal length, may have also an effect on the normal protein folding, due to the substitution of the electrically charged side chain of a glutamate for a glycine with a small nonpolar side chain. It is possible the DEAF1 variants could influence other aspects of DEAF1 function or activity that may not be detected by the cell-based functional assays used in this study. Thus, we predict that these variants constitute a classical, but incomplete, loss of function (hypomorph).

Loss-of-function recessive variants in DEAF1 consist of $\mathrm{p}$. (Arg44Glyfs $\left.{ }^{\star} 25\right)$, p. $\left(\operatorname{Trp} 234^{\star}\right)$, p.(Asp369Alafs $\left.{ }^{\star} 51\right)$, and p. (Cys540Metfs $\left.{ }^{\star} 18\right)$. Only p.(Trp234*) affected transcriptional repression activity. This variant likely results in a truncated protein lacking most of the SAND domain. Our results suggest that the DEAF1 transcript containing the p.(Arg44Glyfs ${ }^{\star} 25$ ) variant can be transcribed using an alternative start codon resulting in a N-terminal deletion of 69 amino acid residues, and thus in an intact SAND domain. Accordingly, these data indicate the MYND domain is not essential for DEAF1 transcriptional repression or activation activity using the in vitro assays in this study. Taken together, the de novo SAND domain variants likely have a dominant-negative effect (antimorph), whereas the biallelic DEAF1 variants lead to a partial loss of function (hypomorph) through either a reduction in function with normal protein levels (missense variants) or reduced protein levels due to NMD (nonsense and frameshift variants). This hypothesis also explains why heterozygous deletions of DEAF1 do not result in a phenotype.

The phenotype did not differ significantly between patients with de novo and biallelic $D E A F 1$ variants, except for the presence of microcephaly $(p<0.0001)$. The latter feature was present in three of the five patients with biallelic variants. Patients with de novo or biallelic DEAF1 variants had DD/ID with severe speech delay, autism, striking mood swings, epilepsy, gait ataxia or other walking problems, a high pain threshold, sleep disturbance, brain abnormalities, and extraneurologic manifestations, such as recurrent infections and gastrointestinal abnormalities that included feeding difficulties, constipation, and/or diarrhea. This suggests that DAND consists of a phenotypic spectrum of features centered around neurodevelopmental delay rather than two distinct clinical syndromes, but a larger number of patients will be required to confirm this hypothesis. To further delineate the clinical spectrum associated with de novo variants in $D E A F 1$, we established a website to collect detailed clinical information of additional individuals to be identified over the coming years (http://humandiseasegenes. nl/deaf1/). As such, the clinical picture may be quite nonspecific. Syndromes to be included in DAND differential diagnosis would be Angelman syndrome, mostly due to ID, gait abnormalities, seizures, paroxysmal bursts of laughter, and fascination with water; Smith-Magenis syndrome, in particular due to the combination of ID and severe sleeping problems; ${ }^{15}$ and Rett syndrome for the biallelic DEAF1 variants, due to the combination of microcephaly and regression. 
The exclusive occurrence of microcephaly and an increased prevalence of some phenotypic abnormalities, such as movement disorder and recurrent structural brain abnormalities, in patients with recessive $D E A F 1$ variants may also suggest that patients with recessive $D E A F 1$ variants may present with a different and somewhat more severe clinical condition than patients with de novo variants, as these variants have a different effect on a molecular level. One exception to the classical DAND phenotype was observed in one patient with biallelic $D E A F 1$ variants as he has preserved cognitive abilities. Nevertheless, this patient with variants p.(Asp369Alafs $\left.{ }^{\star 51}\right)$ and p. (Cys540Metfs $\left.{ }^{\star} 18\right)$ in compound heterozygosity shared clinical features with other patients with biallelic DEAF1 variants, specifically inability to walk or to speak, ${ }^{16,18-20}$ movement disorder, ${ }^{19}$ and minor brain abnormalities including corpus callosum, ventricles, and white matter abnormalities. ${ }^{18,20}$ Another exception to the classical DAND phenotype is the occurrence of normal speech in one patient with the de novo dominant variant p.(Leu214Val), who manifests ID, showing the phenotypic spectrum is wider than previously described.

In conclusion, we propose that different variants in Figure $\mathrm{S} 1$ the DEAF1 gene result in a phenotypic spectrum centered around neurodevelopmental delay. A pathogenic de novo dominant variant would incapacitate the product of the wild-type allele and result in dominant-negative effect, whereas a combination of two recessive variants would result in a partial loss of function. Because the clinical picture can be quite nonspecific, detailed phenotype information, segregation analysis, and functional analysis are fundamental to determine the pathogenicity of novel variants and to improve the care of these patients.

\section{SUPPLEMENTARY INFORMATION}

The online version of this article (https://doi.org/10.1038/s41436019-0473-6) contains supplementary material, which is available to authorized users.

\section{ACKNOWLEDGEMENTS}

We thank the patients and their parents for participating in this study. We are grateful to Claire Thompson, from the Medical Photography Department, Ninewells Hospital and Medical School, Dundee, Angus, UK for taking and providing the clinical photographs of individual AD/3. This work was financially supported by grants from the Dutch Organization for Health Research and Development (ZON-MW grants 917-86-319 and 912-12-109 to B.B.A.d.V.), the Cedars-Sinai Diana and Steve Marienhoff Fashion Industries Guild Endowed Fellowship in Pediatric Neuromuscular Diseases (to T.M.P.), the March of Dimes (grant 6-FY14-422 to M.C.M.), and the National Institutes of Health (grants NINDS 5R21NS091724 to P.J.J. and NINDS R01NS069605 to H.C.M.).

\section{DISCLOSURE}

The authors declare no conflicts of interest.
Publisher's note: Springer Nature remains neutral with regard to jurisdictional claims in published maps and institutional affiliations.

\section{REFERENCES}

1. Vulto-van Silfhout AT, Rajamanickam S, Jensik PJ, et al. Mutations affecting the SAND domain of DEAF1 cause intellectual disability with severe speech impairment and behavioral problems. Am J Hum Genet. 2014;94:649-661.

2. Yip L, Su L, Sheng D, et al. Deaf1 isoforms control the expression of genes encoding peripheral tissue antigens in the pancreatic lymph nodes during type 1 diabetes. Nat Immunol. 2009;10:1026-1033.

3. Barker HE, Smyth GK, Wettenhall J, et al. Deaf-1 regulates epithelial cell proliferation and side-branching in the mammary gland. BMC Dev Biol. 2008;8:94.

4. Gross CT, McGinnis W. DEAF-1, a novel protein that binds an essential region in a Deformed response element. EMBO J. 1996;15:1961-1970.

5. Yip L, Creusot RJ, Pager CT, Sarnow P, Fathman CG. Reduced DEAF1 function during type 1 diabetes inhibits translation in lymph node stromal cells by suppressing Eif4g3. J Mol Cell Biol. 2013;5:99-110.

6. Bottomley MJ, Collard MW, Huggenvik JI, Liu Z, Gibson TJ, Sattler M. The SAND domain structure defines a novel DNA-binding fold in transcriptional regulation. Nat Struct Biol. 2001;8:626-633.

7. Jensik PJ, Huggenvik JI, Collard MW. Identification of a nuclear export signal and protein interaction domains in deformed epidermal autoregulatory factor-1 (DEAF-1). J Biol Chem. 2004;279:32692-32699.

8. Jensik PJ, Huggenvik JI, Collard MW. Deformed epidermal autoregulatory factor-1 (DEAF1) interacts with the Ku70 subunit of the DNA-dependent protein kinase complex. PLOS ONE. 2012;7:e33404.

9. Kateb F, Perrin $H$, Tripsianes $K$, et al. Structural and functional analysis of the DEAF-1 and BS69 MYND domains. PLoS ONE. 2013;8:e54715.

10. Hahm K, Sum EY, Fujiwara $Y$, Lindeman GJ, Visvader JE, Orkin SH. Defective neural tube closure and anteroposterior patterning in mice lacking the LIM protein LMO4 or its interacting partner Deaf-1. Mol Cell Biol. 2004;24:2074-2082

11. Veraksa A, Kennison J, McGinnis W. DEAF-1 function is essential for the early embryonic development of Drosophila. Genesis. 2002;33:67-76.

12. Vissers LE, de Ligt J, Gilissen $C$, et al. A de novo paradigm for mental retardation. Nat Genet. 2010:42:1109-1112.

13. Rauch A, Wieczorek D, Graf E, et al. Range of genetic mutations associated with severe non-syndromic sporadic intellectual disability: an exome sequencing study. Lancet. 2012;380:1674-1682.

14. Wenger AM, Guturu H, Bernstein JA, Bejerano G. Systematic reanalysis of clinical exome data yields additional diagnoses: implications for providers. Genet Med. 2017;19:209-214.

15. Berger SI, Ciccone C, Simon KL, et al. Exome analysis of Smith-Magenislike syndrome cohort identifies de novo likely pathogenic variants. Hum Genet. 2017;136:409-420.

16. Chen L, Jensik PJ, Alaimo JT, et al. Functional analysis of novel DEAF1 variants identified through clinical exome sequencing expands DEAF1associated neurodevelopmental disorder (DAND) phenotype. Hum Mutat. 2017;38:1774-1785.

17. Li SJ, Yu SS, Luo HY, et al. Two de novo variations identified by massively parallel sequencing in 13 Chinese families with children diagnosed with autism spectrum disorder. Clin Chim Acta. 2018;479:144-147.

18. Faqeih EA, Al-Owain M, Colak D, et al. Novel homozygous DEAF1 variant suspected in causing white matter disease, intellectual disability, and microcephaly. Am J Med Genet A. 2014;164A:1565-1570.

19. Rajab A, Schuelke M, Gill E, et al. Recessive DEAF1 mutation associates with autism, intellectual disability, basal ganglia dysfunction and epilepsy. J Med Genet. 2015;52:607-611.

20. Gund C, Powis Z, Alcaraz W, Desai S, Baranano K. Identification of a syndrome comprising microcephaly and intellectual disability but not white matter disease associated with a homozygous c.676C >T p.R226W DEAF1 mutation. Am J Med Genet A. 2016;170A:1330-1332.

21. lafrate $A J$, Feuk $L$, Rivera $M N$, et al. Detection of large-scale variation in the human genome. Nat Genet. 2004;36:949-951.

22. Grimberg J, Nawoschik S, Belluscio L, McKee R, Turck A, Eisenberg A. A simple and efficient non-organic procedure for the isolation of genomic DNA from blood. Nucleic Acids Res. 1989;17:8390.

23. de Ligt J, Willemsen $\mathrm{MH}$, van Bon BW, et al. Diagnostic exome sequencing in persons with severe intellectual disability. N Engl J Med. 2012;367:1921-1929. 
24. Krumm N, Sudmant $\mathrm{PH}, \mathrm{Ko} A$, et al. Copy number variation detection and genotyping from exome sequence data. Genome Res. 2012;22: 1525-1532.

25. Pfundt $R$, Del Rosario $M$, Vissers $L$, et al. Detection of clinically relevant copy-number variants by exome sequencing in a large cohort of genetic disorders. Genet Med. 2017;19:667-675.

26. Jacquemin V, Rieunier G, Jacob $\mathrm{S}$, et al. Underexpression and abnormal localization of ATM products in ataxia telangiectasia patients bearing ATM missense mutations. Eur J Hum Genet. 2012;20:305-312.

27. Livak KJ, Schmittgen TD. Analysis of relative gene expression data using real-time quantitative PCR and the 2(-Delta Delta C(T)) method. Methods. 2001;25:402-408.

28. Ng PC, Henikoff S. SIFT: predicting amino acid changes that affect protein function. Nucleic Acids Res. 2003;31:3812-3814.
29. Adzhubei I, Jordan DM, Sunyaev SR Predicting functional effect of human missense mutations using PolyPhen-2. Curr Protoc Hum Genet. 2013;76:7.20.1-7.20.41. Chapter 7: Unit 720. https://currentprotocols onlinelibrary.wiley.com/doi/abs/10.1002/0471142905.hg0720s76. Accessed 26 March 2019.

30. Kircher M, Witten DM, Jain P, O'Roak BJ, Cooper GM, Shendure J. A general framework for estimating the relative pathogenicity of human genetic variants. Nat Genet. 2014;46:310-315.

31. Lek M, Karczewski KJ, Minikel EV, et al. Analysis of protein-coding genetic variation in 60,706 humans. Nature. 2016;536:285-291.

32. Michelson RJ, Collard MW, Ziemba AJ, Persinger J, Bartholomew B, Huggenvik Jl. Nuclear DEAF-1-related (NUDR) protein contains a novel DNA binding domain and represses transcription of the heterogeneous nuclear ribonucleoprotein A2/B1 promoter. J Biol Chem. 1999;274:30510-30519.

${ }^{1}$ Department of Human Genetics, Radboud University Medical Center, Nijmegen, The Netherlands. ${ }^{2}$ Department of Physiology, Southern Illinois University School of Medicine, Carbondale, IL, USA. ${ }^{3}$ Sheffield Clinical Genetics Service, OPD2 Northern General Hospital, Sheffield, UK. ${ }^{4}$ Department of Clinical Genetics, St George's University Hospitals NHS Foundation Trust \& St George's, University of London, London, UK. ${ }^{5}$ Dartmouth Geisel School of Medicine, Hanover, NH, USA. ${ }^{6}$ Department of Genetics, University Medical Center Utrecht, Utrecht, The Netherlands. ${ }^{7}$ Medical Investigation of Neurodevelopmental Disorders (MIND) Institute, University of California Davis School of Medicine, Sacramento, Sacramento, CA, USA. ${ }^{8}$ Department of Pediatrics, University of California Davis Medical Center, Sacramento, Sacramento, CA, USA. ${ }^{9}$ Department of Clinical Genetics, Nottingham University Hospitals NHS Trust, Nottingham, UK. ${ }^{10}$ Northern Genetics Service, Newcastle upon Tyne Hospitals NHS Foundation Trust, Newcastle upon Tyne, UK. ${ }^{11}$ Sydney Children's Hospital, Randwick, NSW, Australia. ${ }^{12}$ School of Women's and Children's Health, UNSW Medicine, The University of New South Wales, Sydney, NSW, Australia. ${ }^{13}$ Department of Pediatrics, Division of Genetic Medicine, University of Washington-Seattle, Seattle, WA, USA. ${ }^{14}$ Division of Genetic Medicine, Seattle Children's Hospital, Seattle, WA, USA. ${ }^{15}$ Department of Human Genetics, David Geffen School of Medicine at UCLA, Los Angeles, CA, USA. ${ }^{16}$ Division of Medical Genetics, Department of Pediatrics, David Geffen School of Medicine at UCLA, Los Angeles, CA, USA. ${ }^{17}$ Ludwig-Konrad-Str. 14, Bad Wildungen, Germany. ${ }^{18}$ Pediatric Genetics Clinic, Schneider Children's Medical Center of Israel, Petach Tikva, Israel. ${ }^{19}$ Sackler Faculty of Medicine, Tel Aviv University, Tel Aviv, Israel. ${ }^{20}$ Department of Clinical Genetics, Ninewells Hospital and Medical School, Dundee, Angus, UK. ${ }^{21}$ Clinical Genetics, University of Dundee, Dundee, Angus, UK. ${ }^{22}$ Département de Génétique, Hôpital NeckerEnfants Malades, Assistance Publique, INSERM UMR 1163, Institut Imagine, Paris, France. ${ }^{23}$ Département de Génétique, Hôpital Pitié-Salpêtrière, Assistance publique-Hôpitaux de Paris, Paris, France. ${ }^{24}$ Department of Pediatrics, Amsterdam University Medical Centers, Amsterdam, The Netherlands. ${ }^{25}$ North West Thames Genetics NHS, Northwick Park Hospital, London, UK. ${ }^{26}$ Department of Child Neurology, Cortica Healthcare, San Rafael, CA, USA. ${ }^{27}$ Division of Clinical Genetics and Dysmorphology, Department of Pediatrics, Cedars-Sinai Medical Center, Los Angeles, CA, USA. ${ }^{28}$ Department of Pediatrics, Department of Neurology, and the Board of Governors Regenerative Medicine Institute, Cedars-Sinai Medical Center, Los Angeles, CA, USA. ${ }^{29}$ Genetics Research Centre, Molecular and Clinical Sciences Institute, St George's, University of London, London, UK. ${ }^{30} \mathrm{GW}$ Institute for Neuroscience, Department of Pharmacology and Physiology, The George Washington University School of Medicine and Health Sciences, Washington, DC, USA. ${ }^{31}$ Faculty of Medicine "Agostino Gemelli" Catholic University of the Sacred Heart, Rome, Italy. ${ }^{32}$ Center for the Study of Rare Inherited Diseases (CeSMER), Niguarda Ca' Granda Metropolitan Hospital, Milan, Italy. ${ }^{33}$ Service de Génétique Clinique, CLAD-Ouest CHU Rennes, Univ Rennes, CNRS 6290 Institut de Génétique et Développement de Rennes (IGDR), Rennes, France. ${ }^{34}$ Univ Rennes, CHU Rennes, CNRS, IGDR, UMR 6290, Rennes, France. ${ }^{35}$ Division of Human Genetics, Department of Pediatrics, Hasbro Children's Hospital, Warren Alpert Medical School of Brown University, Providence, RI, USA 